\title{
Infrared and Terahertz time-domain imaging for evaluation of impacted thick homogeneous particleboards of sugarcane bagasse
}

\author{
by H. Zhang ${ }^{1,2,+,}$, S. Sfarra ${ }^{3}$, A. Osmann ${ }^{2,4}$, K. Szielasko ${ }^{5}$, C. Stumm ${ }^{5}$, F. Sarasini ${ }^{6}$, Xavier Maldague ${ }^{1}$ \\ ${ }^{1}$ Department of Electrical and Computer Engineering, Computer Vision and Systems Laboratory (CVSL), Laval \\ University, Quebec City, G1V 0A6, Canada \\ ${ }_{2}^{2}$ Department of Inspection of Components and Assemblies, Fraunhofer Institute for Nondestructive Testing IZFP, \\ 66123 Saarbrücken, Germany \\ ${ }^{3}$ Department of Industrial and Information Engineering and Economics, University of L'Aquila, 67100 L'Aquila, \\ Italy \\ ${ }^{4}$ University of Applied Sciences, htw saar, 66117 Saarbrücken, Germany \\ 5 Department of Materials characterization, Fraunhofer Institute for Nondestructive Testing IZFP, 66123 \\ Saarbrücken, Germany \\ ${ }^{6}$ Department of Chemical Engineering Materials Environment \& UDR INSTM, Sapienza University of Rome, \\ 00184 Rome, Italy \\ 3G8, Canada \\ ${ }^{+}$Current address: Department of Mechanical and Industrial Engineering, University of Toronto, Toronto, M5S \\ * Corresponding author: Hai.Zhang.1@ulaval.ca (Hai Zhang)
}

\begin{abstract}
In this paper, both infrared thermography (IRT) and terahertz time-domain imaging (THz-TDI) were used to evaluate impacted homogeneous particleboards of sugarcane bagasse (SCB), which have the thickness of $2 \mathrm{~cm}$. In this study, the THz data were processed by applying the well-known image processing algorithms used in IRT. The comparative results show that IRT can provide some finer details in the detection of shallow thermal imprints; however, it is difficult to reveal deeper (typically $\sim 1 \mathrm{~cm}$ ) information. On the contrary, THz-TDI may explore the entire thickness of the samples, although - for this specific study - its resolution is lower. In addition, THz-TDI has the advantage to detect resin-rich and fibre-rich regions.
\end{abstract}

\section{Introduction}

As a time-domain imaging technique, infrared thermography (IRT) can use many advanced image processing algorithms. Infrared image processing techniques can improve the detection capability of IRT. However, it is impossible to overcome physical restrictions inherent to different factors. The use of the mid-infrared is usually not suitable for the inspection of natural fibres due to the low diffusivity value of the materials. Recently, the emerging terahertz time-domain Imaging (THz-TDI) technique is becoming the most attractive non-destructive testing technique, especially for plant fibre reinforced composites (PFRC).

$\mathrm{THz}$ region of electromagnetic spectrum falls between $100 \mathrm{GHz}$ and $10 \mathrm{THz}$. It lies in the gap between electronic and optical signal generation schemes or, in other words, between microwave and infrared. THz radiation has the capability to penetrate deeper non-metallic materials; it also allows the spectroscopy effect for imaging the inner structures. THz radiation is non-ionizing and non-destructive. In this respect, THz systems can be applied on artworks, integrated circuits, weapons, biomedical and composite materials. The whole set of positivity of $\mathrm{THz}$ imaging systems still remain to be discovered; the same can be said about the advanced image processing techniques linked to them. Similar to IRT, THzTDI is a time-domain imaging technique, too. Therefore, the application of the image processing techniques (usually used for IRT) into the THz-TDI field can be considered as an attractive idea.

In this paper, both IRT and THz-TDI were used to inspect homogeneous particleboards of sugarcane bagasse (SCB) impacted at different energies. Infrared image processing techniques were applied to the THz data. The latter shows that IRT and THz-TDS techniques when combined together can reveal undetectable defects to the naked eye with a different resolution due to the depth probed, the equipment used, the physical constraints, etc.

\section{Specimens}

The high-density homogeneous particleboards of SCB and polyurethane resin are based on castor oil, assuming a nominal density of $800 \mathrm{~kg} / \mathrm{m}^{3}$ and dimension of $0.55 \times 0.55 \times 0.016 \mathrm{~m}$. Initially, the SCB was oven dried at a temperature of $60 \mathrm{C}$ for $24 \mathrm{~h}$ to reduce the moisture content to around $12 \%$. After drying, it was sieved in a sieve shaker. The bagasse particles retained in the sieve mesh with openings greater than $2 \mathrm{~mm}$ were milled in a knife mill to produce particles of size 
up to $8 \mathrm{~mm}$. The particles with a size lower than $2 \mathrm{~mm}$ were removed from the manufacturing process because they are not suitable for the step of dispersing the resin. Subsequently, the particles of SCB were introduced in a planetary mixer and the adhesive polyurethane resin based on castor oil was added, in a proportion of $15 \%$ by weight of the dry mass of bagasse, for better uniformity in the distribution of the adhesive in particles. After this mixing period, the particles were introduced into a mattress forming mold, with dimensions of $0.55 \times 0.55 \mathrm{~m}$ and pressed with a pressure of $5 \mathrm{MPa}$ at a temperature of $100 \mathrm{C}$, for a period of $10 \mathrm{~min}$. At the end of the pressing process, the complete curing process of the resin required a period of $72 \mathrm{~h} \mathrm{[1]}$.

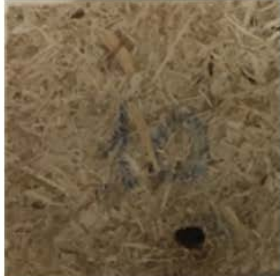

(a) $5 \mathrm{~J}$

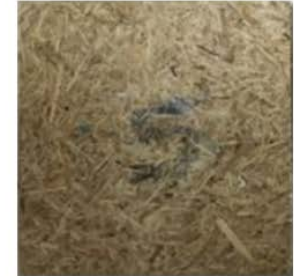

(b) $10 \mathrm{~J}$

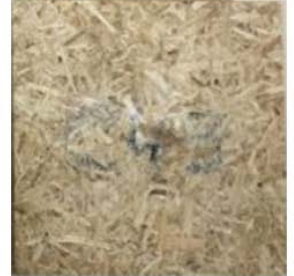

(c) $20 \mathrm{~J}$

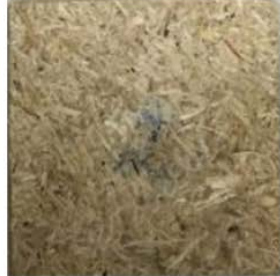

(d) $30 \mathrm{~J}$

Fig. 1: Photographs of the impacted SCB specimens [1].

Low velocity impact tests at four different energy levels, namely 5, 10, 20 and $30 \mathrm{~J}$ (see Figure 1) were carried out using an instrumented drop-weight impact testing machine (CEAST/Instron 9340). A steel mass was attached to the steel impactor with a hemispherical tip of $20 \mathrm{~mm}$ diameter for a total weight of $3 \mathrm{~kg}$. The different energy levels were obtained by changing the height of release of the impactor. The specimens were clamped circumferentially along a diameter of $40 \mathrm{~mm}$ in a pneumatic-actuated clamping fixture. During the test, the time histories of impact force and the initial impact velocity were recorded. Post-impact, the permanent dent depth of each coupon was measured using a noncontact profilometer (Taylor-Hobson Talyscan 150) with a scanning speed of 10,500 um/s and a nominal vertical resolution of $1 \mathrm{um}$. The dent depth as a function of impact energy is shown in Table 1 [1].

Table 1. Dent depth as a function of impact energy [1]

\begin{tabular}{lcccc}
\hline Impact energy $(\mathrm{J})$ & 5 & 10 & 20 & 30 \\
\hline Dent depth $(\mu \mathrm{m})$ & 592 & 1128 & 1469 & 1545 \\
\hline
\end{tabular}

\section{Methodology}

\subsection{Infrared thermography}

As an optical excitation thermographic approach, pulsed thermography (PT) uses high-energy lamps to generate a uniform heating on an object surface. An infrared camera is used to record the surface temperature profile. In PT, a subsurface defect causes a non-uniform cooling, which can be identified in an imaging sequence. As a time-domain sequenced imaging approach, PT allows the application of the advanced imaging processing techniques to obtain more visible imprints of the defects [2].

Figure 2 shows the schematic configuration for PT. Two halogen lamps (OMNLUX PAR64,1000W) were used to generate the pulsed excitation signal. A mid-wave infrared camera (FLIR Phoenix, InSb, 3-5 um, $640 \times 512$ pixels) at a frame rate of $55 \mathrm{~Hz}$ was used to record the temperature profile. The heating time was $5 \mathrm{~s}$ and the cooling time was $35 \mathrm{~s}$. 


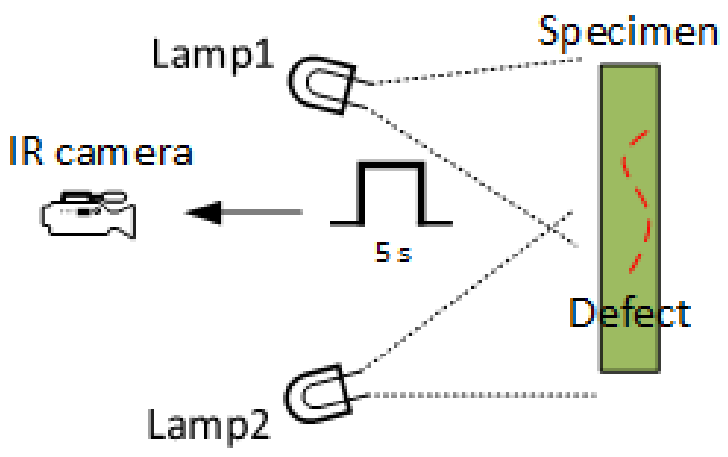

Fig. 2: Pulsed thermography schematic configuration [2].

\subsection{Terahertz time-domain imaging}

Fig. 2 shows the schematic configuration of the employed THz-TDI system. The pump wavelength was $1550 \mathrm{~nm}$, and the repetition rate was $80 \mathrm{MHz}$. The system has a $5 \mathrm{GHz}$ frequency resolution. The experiments were performed in transmission mode. The specimens were located in the focus of the optical system between the $\mathrm{THz}$ emitter and the receiver. A main advantage of transmission mode is that the complex refractive index and the attenuation coefficient of a measured material can be easily determined [3].

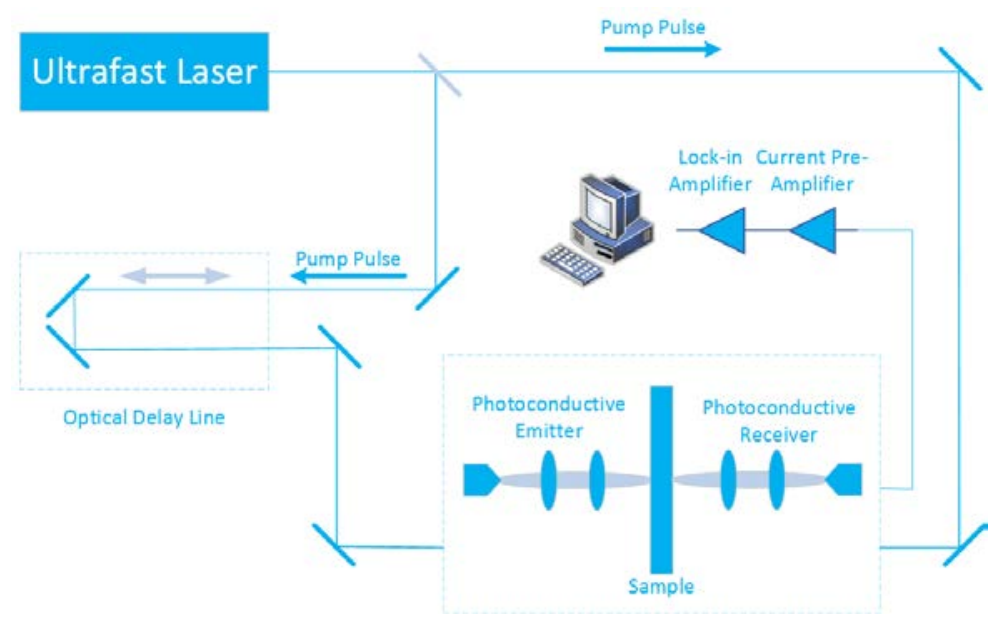

Fig. 3: Terahertz time-domain imaging schematic configuration [3]

\section{Results and discussion}

Fig. 4 shows the PT results of the specimens. Pulsed phase thermography (PPT) and principle component thermography (PCT) were used to process the thermal data. It can be observed that the impact damage can be slightly detected from the front side of the impact. However, the impact damage cannot be inspected from the back side of the impact due to the fact that the specimens are very thick $(2 \mathrm{~cm})$ for infrared thermography. The fibres are shown clearly thanks to the high resolution of the applied infrared camera. 
$5 \mathrm{~J}$

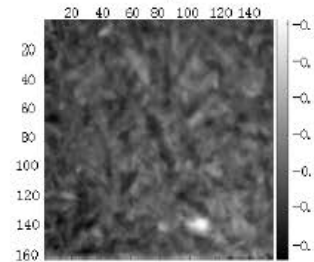

(a)

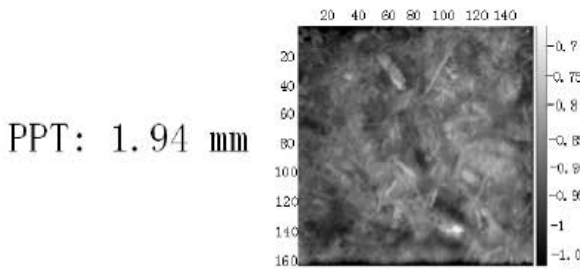

(e)

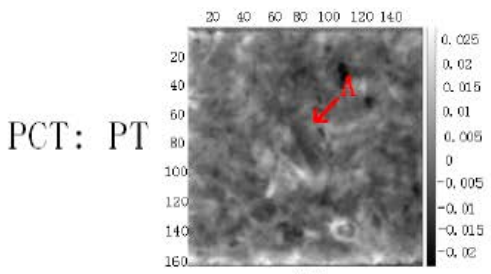

(i)
$10 \mathrm{~J}$

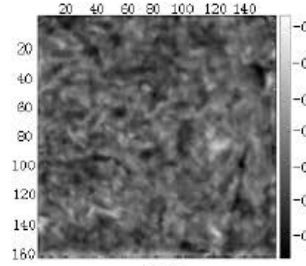

(b)

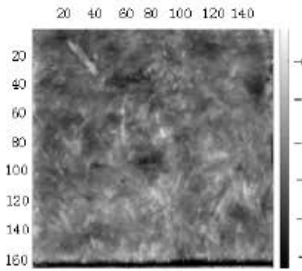

(f)

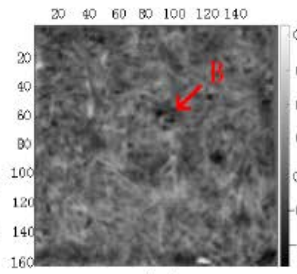

(j)
$15 \mathrm{~J}$

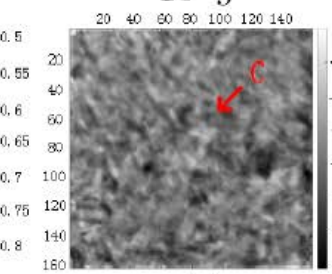

(c)

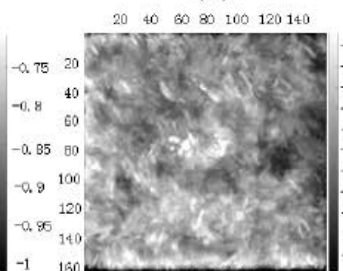

$(\mathrm{g})$

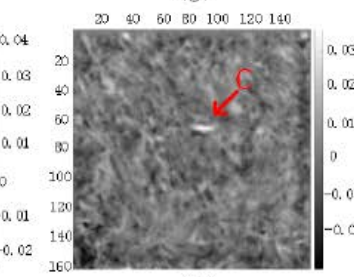

(k)
$20 \mathrm{~J}$

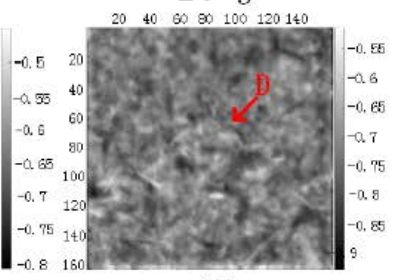

(d)

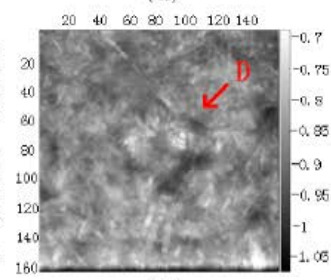

(h)

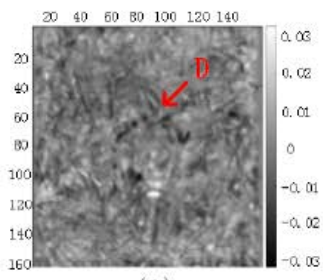

(1)

Fig. 4: Pulsed thermography results [1].

Fig. 5 shows the THz-TDI results, which are obtained using a peak-to-peak imaging method. This method is often used in THz-TDI, which usually can show defects easily. However, it is observed that the impact damage is not inspected in peak-to-peak imaging. This is due to the fact that the impact damage does not provide a significant peak signal difference from the unimpacted areas. However, the resin-rich and resin-poor regions can be inspected easily due to the different absorption coefficients of different materials. This is a significant advantage of THz-TDI.

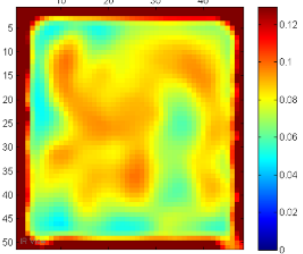

SCB \#1

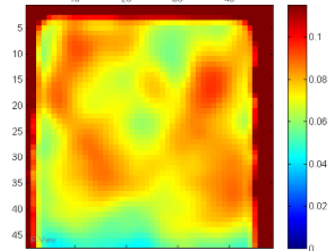

SCB \#2

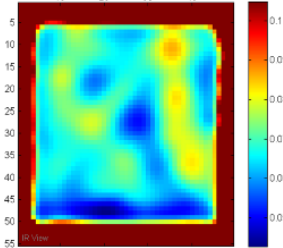

SCB \#3

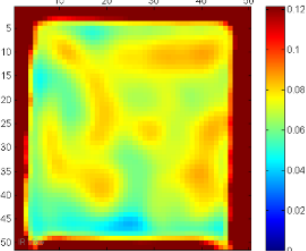

SCB \#4

Fig. 5: Terahertz time-domain imaging results: peak-to-peak.
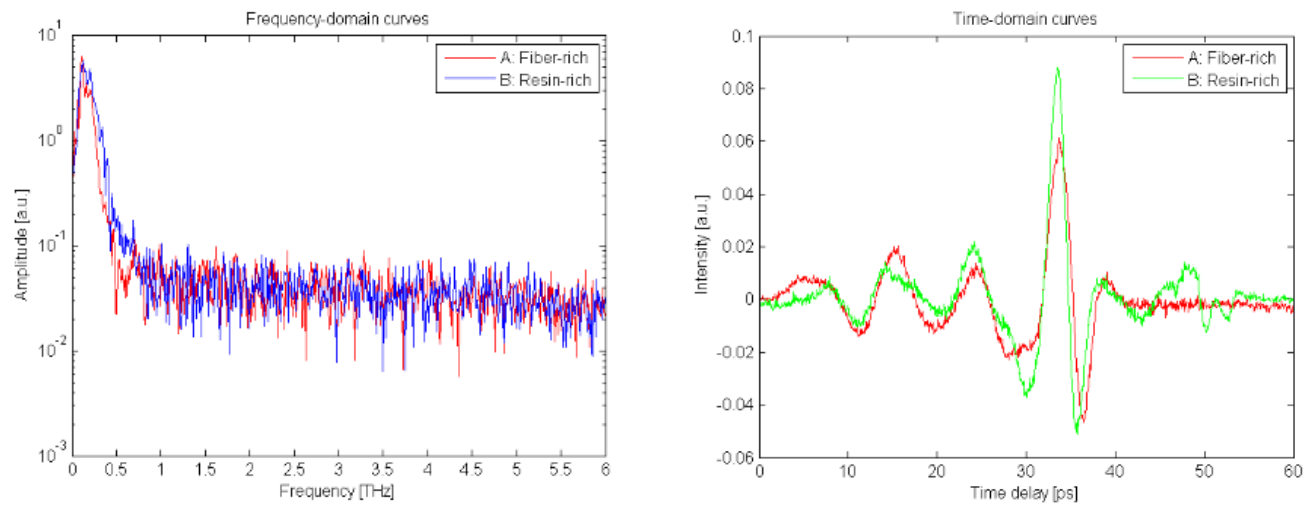
(a)

(b)

Fig. 6: Terahertz analytical curves: (a) in frequency-domain, and (b) in time-domain.

Figs. $6 \mathrm{a}$ and $6 \mathrm{~b}$ show the frequency-domain and the time-domain curves, respectively. They are linked to a fibrerich zone and a resin-rich zone.

Advanced image processing algorithms may enhance the THz-TDI images and achieve more detective details. In this work, PPT was also used in THz-TDI to achieve the frequency-domain images. Fig. 7 shows the results after PPT processing, which involves $0.1 \mathrm{THz}$ and $0.2 \mathrm{THz}$. It can be observed that fiber-rich and resin-rich regions can also de detected in the amplitude results, while it is not obvious in the phase results. Nevertheless, the impact damage cannot be inspected in these THz frequency domains. This is because the specimens are very thick $(2 \mathrm{~cm})$, and a high frequencydomain imaging will lose the impact features due to the attenuation of signals. Therefore, a low frequency-domain imaging might provide more information.

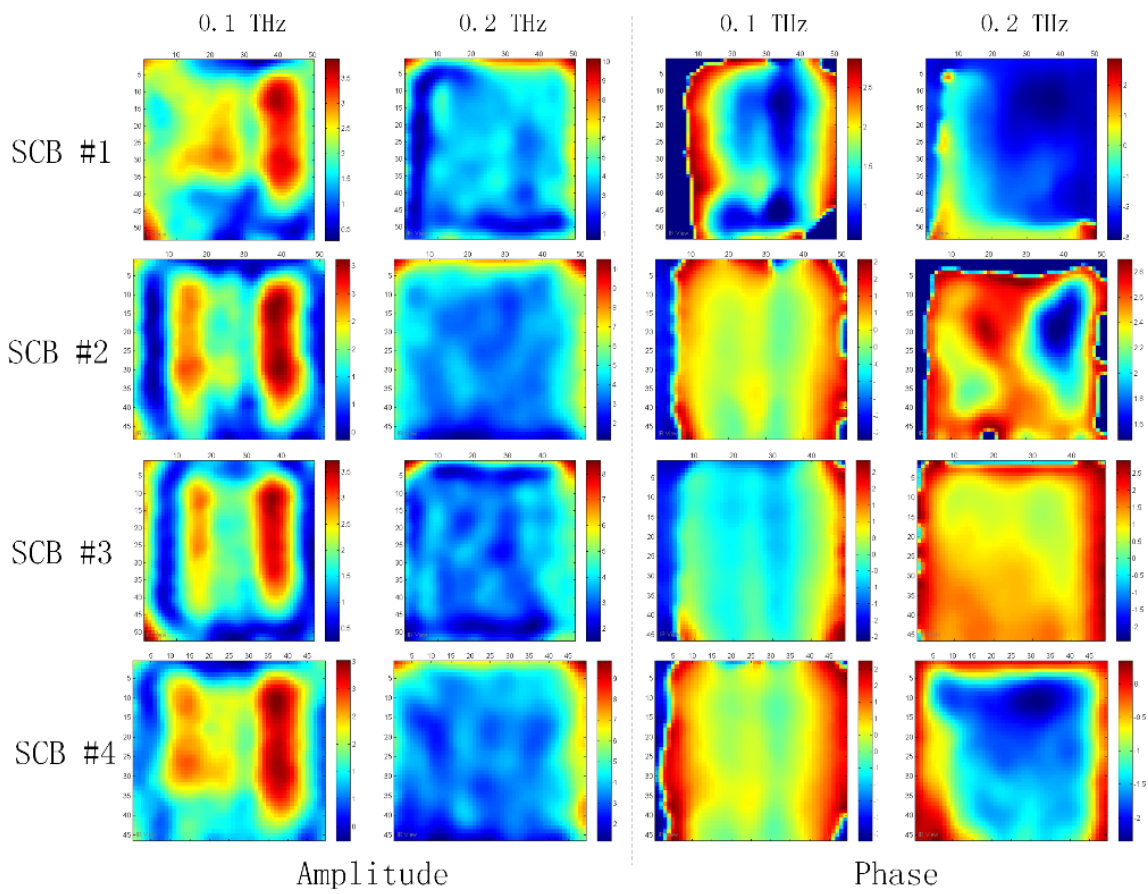

Fig. 7 PPT applied on terahertz time-domain imaging: 0.1THz and 0.2 THz.

Fig. 8 shows the THz-TDI results after PPT processing, which involves $0.02 \mathrm{THz}$ and $0.03 \mathrm{THz}$. The impact damages can be observed in these frequencies in both amplitude and phase imaging. However, amplitude images can provide more details due to their higher resolution. It is worth mentioning a fact that the resolution of THz-TDI is lower than $\mathrm{PT}$ in this work. This is because the resolution limitation of the applied system. However, new systems with much better resolution are available now and they can provide as good resolution as infrared. In addition, THz-TDI works better for thick samples than PT thanks to its stronger penetration capability. 


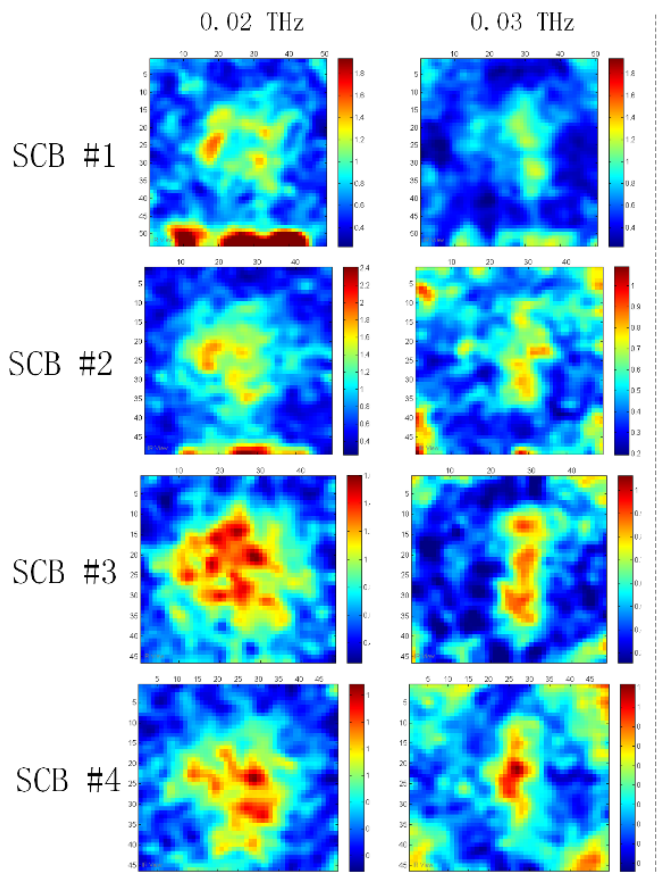

Amplitude
$0.02 \mathrm{TH} \%$
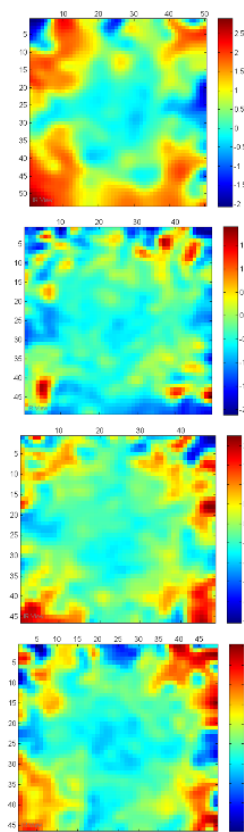

Phase
$0.03 \mathrm{THz}$
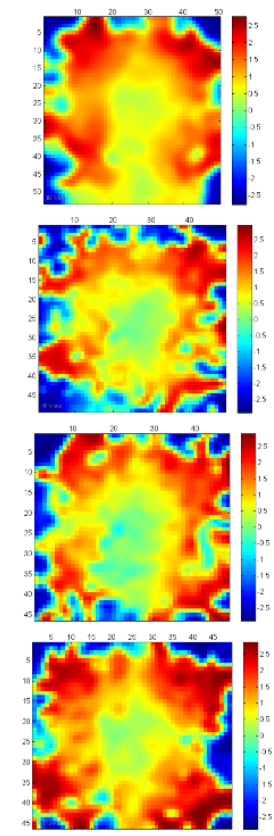

Fig. 8 PPT applied on terahertz time-domain imaging: 0.02THz and 0.03 $\mathrm{THz}$.

\section{Conclusions}

THz-TDI can be used to detect thick SCB materials due to its stronger penetration capability than PT. The resolution is limited by the system in this work, but a much higher resolution system is available now. THz-TDI should provide as good resolution as PT. PT can provide clear details for shallow depth, but it is difficult for deeper depth when considering thick materials. In addition, THz-TDS transmission mode is sensitive to resin-rich and fibre-rich regions due to the absorption coefficients of different materials, which is a specific advantage of THz-TDI

\section{REFERENCES}

[1] Zhang, Hai, et al. "Thermographic Non-Destructive Evaluation for Natural Fiber-Reinforced Composite Laminates." Applied Sciences 8.2 (2018): 240.

[2] Zhang, Hai, et al. "Enhanced Infrared Image Processing for Impacted Carbon/Glass Fiber-Reinforced Composite Evaluation." Sensors 18.1 (2017): 45.

[3] Zhang, Hai, et al. "Terahertz Image Improvement for An Environmentally Friendly Sandwich Structure." CCECE (2018). 